\title{
Assessment of exposure to environmental tobacco smoke in young adolescents following implementation of smoke-free policy in Italy
}

\author{
M. Pellegrini ${ }^{\text {a }}$, M.C. Rotolo ${ }^{a}$, S. La Grutta ${ }^{\text {b }}$, F. Cibella ${ }^{\text {b }, ~ O . ~ G a r c i a-A l g a r ~}{ }^{c}$, A. Bacosi ${ }^{\text {a }}$, G. Cuttitta $^{\text {b }}$, \\ R. Pacifici ${ }^{a}$, S. Pichini ${ }^{\text {a,* }}$ \\ ${ }^{a}$ Department of Therapeutic Research and Medicines Evaluation, Istituto Superiore di Sanitá, V.le Regina Elena 299, 00161 Rome, Italy \\ ${ }^{\mathrm{b}}$ Consiglio Nazionale delle Ricerche, Istituto di Biomedicina e Immunologia Molecolare, Palermo, Italy \\ ${ }^{\mathrm{c}}$ Unitat de Recerca Infància i Entorn (URIE), Paediatric Service, IMIM-Hospital del Mar, Barcelona, Spain
}

\section{A R T I C L E I N F O}

\section{Article history:}

Received 17 June 2009

Accepted 5 August 2009

Available online 13 January 2010

\section{Keywords:}

Environmental tobacco smoke

Hair nicotine

Urinary cotinine

\begin{abstract}
A B S T R A C T
We investigated acute and chronic exposure to environmental tobacco smoke (ETS) in a cohort of young adolescents using urinary cotinine and hair nicotine testing after recent implementation of Italian smoke free legislation.

Study subjects were 372 Italian young adolescents, between 10 and 16 years of age from the principal city of Sicily, Palermo. Urine and hair samples were collected between November 2005 and May 2006, when the legislation to ban smoking in all the enclosed places of employment (including bars, restaurants, pubs) was completely enforced. An exhaustive questionnaire including sociodemographic characteristics and active and passive exposure to cigarette smoking was completed. Urinary cotinine was analyzed by radioimmunoassay and hair nicotine by a validated GC/MS method.

Based on urinary cotinine results, $2.1 \%$ and $89 \%$ of the study participants, respectively, showed nonexposure and low acute exposure to ETS, whereas only $1.6 \%$ presented very high exposure or a hidden active smoking habit in the recent past. Hair nicotine disclosed non-exposure and low exposure to ETS in $11.8 \%$ and $65.6 \%$ of the young adolescents, respectively, taking into consideration a larger time-window. High repeated exposure, suggesting active smoking in some cases was observed in $8.6 \%$ of the study subjects. Hair nicotine was inversely related to educational level of the adolescents' parents.

Overall, due to the implementation of smoke-free legislation and information campaign against smoking, a significant trend toward low exposure to ETS was observed in this study cohort with no association between exposure to ETS and respiratory illnesses.
\end{abstract}

(c) 2009 Elsevier Ireland Ltd. All rights reserved.

\section{Introduction}

The scientific community agrees that tobacco smoking is the main cause of morbidity and avoidable mortality today [1]. Prevention of smoking and the fight against tobacco consumption are within the primary objectives of the 21 st century health policies in the international community and in our country [1-3].

Italy has always been among the countries more involved in the reduction of exposure to environmental tobacco smoke, reduction of tobacco use initiation and the increase of tobacco use cessation. Particularly, the government has always been involved in tobacco control through legislation promoting smoke free environment as a recommended strategy to reduce smoking prevalence and subsequently environmental tobacco smoke in the community.

\footnotetext{
* Corresponding author. Tel.: +39 06 49903682; fax: +39 0649902016.

E-mail address: simona.pichini@iss.it (S. Pichini).
}

Since 1975, several laws regarding the prohibition of smoking in public places (hospitals, schools, libraries, cinemas workplaces of public administration) were passed [4,5]. Finally, thanks to a law passed in January 2003 regarding the "Protection of non-smoker's health", smoking has been forbidden in all the enclosed places of employment (including bars, restaurants, pubs) with the exception of those places with rooms reserved to smokers' [6]. It took all of 2004 for the law to be extensively applied and it has been completely enforced by 2005.

At the same time, starting in 2000, several information campaigns and educational actions against smoking have been implemented in Italy by the Health Policy Authorities and the National Institute of Health [7]. A free telephone counselling line has been created to help and inform smokers and their families. Clinical guidelines to help smokers quit the habit have been produced and distributed to all health professionals and family doctors from the National Health System [8].

Involuntary exposure of children and young adolescents to environmental tobacco smoke (ETS) is an important and 
preventable cause of morbidity. Evidence from meta-analyses and systematic reviews on the subject show that ETS increases the risk of several childhood illnesses (e.g. sudden infant death syndrome, middle ear disease, respiratory tract infections) and of producing carcinogen-protein adducts in children and young adolescents, which in turn may be considered as a potential risk of cancer later in life $[9,10]$.

Since the last decade hair testing for nicotine and cotinine have been used as a complement to urinary cotinine for objectively assessing the exposure to cigarette smoke with a longer timewindow since hair testing accounts for a mean repeated exposure whereas urine testing provides information on acute exposure in the days immediately prior to analysis [11].

We aimed to evaluate recent and past exposure to ETS in an urban cohort of young adolescents in light of the recent laws and information campaigns against smoking, which came into force in Italy.

Self-reported questionnaire has been used to assess active and passive exposure to tobacco smoke and respiratory health in the participating youngsters, and urinary cotinine and hair nicotine and cotinine were measured as biomarkers of short and long term exposure to tobacco smoke.

\section{Materials and methods}

\subsection{Subjects}

A cross-sectional study was conducted in a sample of schoolchildren, aged 10-16 years, from junior high schools of Palermo (680,000 inhabitants), Sicily, an island in Southern Italy, in the period November 2005-May 2006. A number of 372 young adolescents were randomly selected and were completely evaluated by questionnaire, lung function testing and skin prick test (SPT) at school. No subject declared to be an active smoker. All subjects provided urine and hair samples. Signed consent was obtained from the youngsters' parents and the study was approved by the local ethics committee.

Urine samples were collected at the time of clinical evaluation, and immediately stored at $-20^{\circ} \mathrm{C}$. Hair samples were cut at the scalp in the vertex region and stored at ambient temperature in a paper bag.

\subsection{Urine and hair samples analysis}

Urinary cotinine was analyzed in duplicate using a double antibody radioimmunoassay according to a method described previously [12]. The level of crossreactivity of the cotinine antibody with other nicotine metabolites was less than $5 \%$. The limit of detection was $0.2 \mathrm{ng} / \mathrm{mL}$ and the inter-assay coefficient of variation ranged between $6 \%$ and $10 \%$. The proximal $4 \mathrm{~cm}$ hair segment, accounting for approximately the last 4 months of exposure to ETS, was analyzed. Hair nicotine and cotinine were detected by a validated liquid chromatography-mass spectrometry (LC-MS) assay, presenting limits of quantification of 0.2 and $0.1 \mathrm{ng} / \mathrm{mg}$ hair for nicotine and cotinine, respectively [13].

\subsection{Statistical analysis}

The young adolescents were classified as non-exposed $(<1 \mathrm{ng} / \mathrm{ml})$, low $(1.1-$ $14 \mathrm{ng} / \mathrm{ml}$ ), medium (14.1-50 ng/ml), high and very high ( $>50.1 \mathrm{ng} / \mathrm{ml}$ ) exposure to ETS using urinary cotinine concentration ranges, as previously reported [14].

Of hair nicotine and cotinine, only hair nicotine was considered to discriminate between different ranges of long-time window of exposure to ETS. Similar to previous reports [11], subjects were classified as non-exposed $(<0.2 \mathrm{ng} / \mathrm{mg}$ hair nicotine), low (0.2-4 ng/mg hair nicotine), medium (4.1-9 ng/mg hair nicotine), high and very high $(>9.1 \mathrm{ng} / \mathrm{mg}$ hair nicotine) exposure to ETS.

One-way (ANOVA) and frequency distribution tables $\left(\chi^{2}\right)$ for parametric variables were performed. Since the distribution of hair nicotine values was highly skewed, analysis was performed after natural log transformation.

All computations were performed by StatView statistical software package (SAS Institute, Cary, NC, USA). A probability level of $p<0.05$ was selected as statistically significant.

\section{Results and discussion}

According to urinary cotinine values and the international cutoffs to distinguish between different levels of exposure to ETS, the majority of young adolescents (91.1\%) presented as non-exposed or at low exposure to ETS in the recent past, whereas less than $2 \%$
Table 1

Classification of young adolescents' recent exposure to ETS according to urinary cotinine levels.

\begin{tabular}{lcrr}
\hline & $\begin{array}{l}\text { Urine cotinine } \\
(\mathrm{ng} / \mathrm{ml})\end{array}$ & \multicolumn{1}{c}{ No. } & $\%$ \\
\hline Non-exposure & $<1$ & 8 & 2.1 \\
Low exposure & $1.1-14$ & 333 & 89.0 \\
Medium exposure & $14.1-50$ & 25 & 6.7 \\
High and very high exposure & $>50.1$ & 6 & 1.6 \\
\hline
\end{tabular}

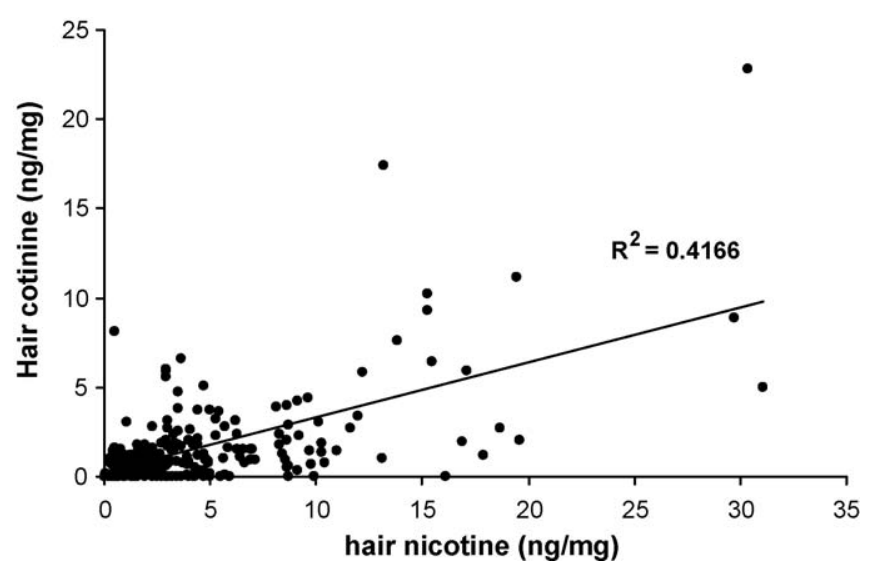

Fig. 1. Values of hair nicotine and hair cotinine in the proximal $4 \mathrm{~cm}$ hair segment for the 372 study subjects.

were highly exposed or suspect of active smoking (Table 1 ). These data, which represent only a short time-interval of exposure (urinary cotinine accounts for the previous 1 or 2 days of exposure) scarcely reflect the results of self-reported questionnaire with the exception of the medium exposure. Indeed of the 372 examined children, $51.2 \%$ declared to be not-exposed to ETS, $9.7 \%$ to be at a low exposure, $6.3 \%$ at medium exposure and finally 32.8 at high and very high exposure.

Nor were these self-reports in accordance with biomarkers accounting for long time window of exposure assessed by nicotine and cotinine measured in hair samples from the subjects. The $4 \mathrm{~cm}$ hair segment was chosen since it was the maximum hair strand length available for all the samples. As shown in Fig. 1, the values found for the two biomarkers scarcely correlated and many values obtained for hair cotinine were under the analytical limit of quantification. For statistical purpose, the value for hair cotinine used in these situations was zero.

The poor correlation between hair nicotine and cotinine, already reported by our and other study groups $[15,16]$, has been explained by different rates of incorporation of the two substances in the hair shaft and by the subsequent partial removal of environmental nicotine by organic washings prior to LC-MS analysis [17].

To discriminate between different levels of long-time window of exposure to ETS it was decided to use hair nicotine with arbitrary cut-offs established previously [11] According to hair nicotine,

Table 2

Classification of young adolescents' long-term exposure to ETS according to hair nicotine levels.

\begin{tabular}{llrr}
\hline & $\begin{array}{l}\text { Hair nicotine } \\
(\mathrm{ng} / \mathrm{mg})\end{array}$ & No. & $\%$ \\
\hline Non-exposure & $<0.2$ & 44 & 11.8 \\
Low exposure & $0.2-4$ & 125 & 65.6 \\
Medium exposure & $4.1-9$ & 52 & 13.9 \\
High and very high exposure & $>9.1$ & 28 & 8.6 \\
\hline
\end{tabular}


Table 3

Characteristics of the study population according to hair nicotine.

\begin{tabular}{|c|c|c|c|c|c|}
\hline & Non-exposure to ETS & Low exposure to ETS & Medium exposure to ETS & $\begin{array}{l}\text { High and very } \\
\text { exposure to ETS }\end{array}$ & $p$-Value \\
\hline \multicolumn{6}{|l|}{ Anthropometric characterisitcs } \\
\hline Age (mean $\pm \mathrm{SD}$ ) & $12.7( \pm 0.61)$ & $12.0( \pm 0.73)$ & $12.3( \pm 0.79)$ & $12.4( \pm 0.67)$ & $<0.0001^{\mathrm{a}, *}$ \\
\hline $\operatorname{Sex}(M / F)$ & $26 / 18$ & $121 / 123$ & $20 / 32$ & $18 / 14$ & $0.19^{\mathrm{b}}$ \\
\hline Height $(\mathrm{cm})$ & $156.3( \pm 7.1)$ & $151.2( \pm 7.7)$ & $153.8( \pm 7.5)$ & $151.8( \pm 8.2)$ & $0.0004^{\mathrm{a}, *}$ \\
\hline Weight (kg) & $54.2( \pm 12.5)$ & $47.5( \pm 11.8)$ & $51.5( \pm 14.0)$ & $47.4( \pm 10.2)$ & $0.0028^{\mathrm{a}^{*}}$ \\
\hline \multicolumn{6}{|l|}{ Respiratory illnesses } \\
\hline Asthma ever (\%) & 22.7 & 21.7 & 17.3 & 25.0 & $0.84^{\mathrm{b}}$ \\
\hline Current asthma (\%) & 13.6 & 9.0 & 7.7 & 6.3 & $0.68^{\mathrm{b}}$ \\
\hline Rhinoconjubctivitis (\%) & 43.2 & 32.0 & 34.6 & 31.3 & $0.53^{\mathrm{b}}$ \\
\hline Wheeze in the last 12 months & 15.9 & 24.2 & 25.0 & 18.8 & $0.60^{\mathrm{b}}$ \\
\hline Nocturnal cough (\%) & 38.6 & 38.9 & 40.4 & 46.9 & $0.85^{\mathrm{b}}$ \\
\hline Chronic cough (\%) & 25.0 & 25.0 & 25.0 & 21.9 & $0.98^{\mathrm{b}}$ \\
\hline
\end{tabular}

a ANOVA.

b $\chi^{2}$.

Statistically significant; $p<0.05$.

$11.8 \%$ of the study participants presented as not exposed to ETS, $65.6 \%$ at low exposure to ETS, $13.9 \%$ at medium exposure and finally $8.6 \%$ at high and very high exposure (Table 2 ). These results correlated only in part with those obtained for urinary cotinine. This was not unexpected since urinary cotinine was not collected at the steady state (first urine of the morning) [18], and as already demonstrated it represents a short-term, acute and irregular exposure to ETS [19], whereas hair nicotine (or cotinine) accounts for a long-term mean cumulative exposure to ETS.

Nonetheless, in general terms, the objective measures of exposure to ETS indicate that the majority of young adolescents underwent a low exposure (or no exposure at all) to ETS, even when they considered themselves as exposed to smoking. Another important encouraging result of this study is that high and very high cumulative exposure, which can be also due to hidden active smoking, was present in less than $10 \%$ of the study population in contrast with the data of the National Survey on Smoking which reports $19.8 \%$ active smokers under the age of 16 [20] (Table 3).

Surprisingly, the most ETS exposed adolescents were younger, shorter and with a lower weight when compared with the nonexposed adolescents. It is worth mentioning that, this extended low exposure in the studied population did not produce an association between exposure to ETS and respiratory illnesses, probably related to other risk factors [21] (Table 3).

Finally, the study confirmed what it has been already demonstrated in other cohorts [9,16], that young adolescents more exposed to ETS belong to families with a lower educational level (Fig. 2).



Fig. 2. Parent's education level was classified as follows: 0: primary and secondary schools; 1 : high school; 2 : degree, post degree ("statistically significant, $p<0.05$ ).
Unfortunately, a limitation of this study is that biomarkers of short and long-term exposure to ETS were used for the first time in a population of young adolescents from Sicily, Italy and no comparison with past relevant data can de performed.

In conclusion, due to the implementation of smoke-free legislation and information campaign against smoking, a significant trend toward low exposure to ETS was observed in this study cohort resulting in no association between exposure to ETS and respiratory illnesses.

\section{References}

[1] J. Cornuz, J.P. Zellweger, B. Burnard, Smoking cessation: importance for the patient and role of the practitioner, Schweiz. Med. Wochenschr. 124 (1994) 1315-1325.

[2] Available at www.ministerosalute.it (accessed January 25, 2007).

[3] Available at www.surgeongeneral.gov/library/tobacco use/ (accessed February 13, 2007).

[4] Law 11 November 1975 n. 584, Article 1 Gazzetta Ufficiale no. 322, 5 December 1975.

[5] DPCM 14 December 1995 Gazzetta Ufficiale, 15 January 1996, n. 11.

[6] Law 16 January 2003 n. 3 Article 51, Gazzetta Ufficiale n. 15del 20 Gennaio 2003 Supplemento Ordinario n. 5.

[7] A. Di Pucchio, E. Pizzi, G. Carosi, M. Mazzola, D. Mattioli, R. Pacifici, S. Pichini, National survey of the smoking cessation services in Italy, Int. J. Environ. Res. Public Health 6 (2009) 915-926.

[8] E. Pizzi, A. Di Pucchio, L. Mastrobattista, R. Solimini, R. Pacifici, S. Pichini, A helpline telephone service for tobacco related issues: the Italian experience, Int. J. Environ. Res. Public Health 6 (2009) 900-914.

[9] J.R. DiFranza, C.A. Aligne, M. Weitzman, Prenatal and postnatal environmental tobacco smoke exposure and children's health, Pediatrics 113 (2004) 1007-1015.

[10] J. Bartsch, E. Hictanen, The role of individual susceptibility in cancer burden related to environmental exposure, Environ. Health Perspect. 104 (1996) 569577.

[11] S. Pichini, Ó. García-Algar, R. de la Torre, in: P. Kintz (Ed.), Clinical Applications of Hair Analysis. Analytical And Practical Aspects of Drug Testing in Hair, 2006, 201222.

[12] H. Van Vunakis, H.B. Gijka, J.J. Langone, Radioimmunoassay for nicotine and cotinine, IARC Sci. Publ. 109 (1993) 293-299.

[13] F. Vagnarelli, S. Amarri, G. Scaravelli, M. Pellegrini, O. Garcia-Algar, S. Pichini, TDM grand rounds: neonatal nicotine withdrawal syndrome in an infant prenatallyand postnatally exposed to heavy cigarette smoke, Ther. Drug Monit. 28 (2006) 585588.

[14] C. Puig, O. Garcia-Algar, T. Monleon, R. Pacifici, P. Zuccaro, J. Sunyer, C. Figueroa, S. Pichini, O. Vall, A longitudinal study of environmental tobacco smoke exposure in children: parental self reports versus age dependent biomarkers, BMC Public Health 8 (2008) 47.

[15] S. Pichini, R. Pacifici, I. Altieri, A.R. Passa, M. Rosa, P. Zuccaro, Analysis of nicotine and cotinine in human hair by high-performance liquid chromatography and comparative determination with radioimmunoassay, in: N.I.D.A. Research Monograph "Hair Testing for Drugs of Abuse: International Research on Standards and Technology", 1995, pp. 212-224.

[16] C. Eliopoulos, J. Klein, D. Chitayat, M. Greenwald, G. Koren, Nicotine and cotinine in maternal and neonatal hair as markers of gestational smoking, Clin. Invest. Med. 19 (1996) 231-242.

[17] E. Jacqz-Aigrain, D. Zhang, G. Maillard, D. Luton, J. André, J.F. Oury, Maternal smoking during pregnancy and nicotine and cotinine concentrations in maternal and neonatal hair, BJOG 109 (2002) 909-911. 
[18] M. Rosa, R. Pacifici, I. Altieri, S. Pichini, G. Ottaviani, P. Zuccaro, How the steadystate cotinine concentration in cigarette smokers is directly related to nicotine intake, Clin. Pharmacol. Ther. 52 (1992) 324-329.

[19] W.K. Al-Delaimy, J. Crane, A. Woodward, Is the hair nicotine level a more accurate biomarker of environmental tobacco smoke exposure than urine cotinine? J. Epidemiol. Commun. Health 56 (2002) 66-71.
[20] www.iss.it/ofad/ (accessed June 2009).

[21] G. Di Lorenzo, A. Drago, M.E. Pellitteri, G. Candore, A. Colombo, M. Potestio, A. Di Salvo, S. Mansueto, C. Caruso, Serum levels of soluble CD23 in patients with asthma or rhinitis monosensitive to Parietaria. Its relation to total serum IgE levels and eosinophil cationic protein during and out of the pollen season, Allergy Asthma Proc. 20 (1999) 119-125. 